\title{
Le rapport d'enseignants du collégial québécois à la lecture d'une œuvre complète : le plan didactique
}

Julie Babin et Olivier Dezutter

\section{(Q) OpenEdition}

\section{Journals}

Édition électronique

URL : http://journals.openedition.org/trema/4649

DOI : $10.4000 /$ trema.4649

ISSN : 2107-0997

Éditeur

Faculté d'Éducation de l'université de Montpellier

Édition imprimée

Date de publication : 1 octobre 2018

Pagination : $17-27$

ISBN : 979-10-96627-05-9

ISSN : 1167-315X

Référence électronique

Julie Babin et Olivier Dezutter, « Le rapport d'enseignants du collégial québécois à la lecture d'une œuvre complète : le plan didactique », Tréma [En ligne], 49 | 2018, mis en ligne le 15 octobre 2018, consulté le 22 avril 2019. URL : http://journals.openedition.org/trema/4649; DOI : 10.4000/

trema.4649

Ce document a été généré automatiquement le 22 avril 2019

Trema 


\section{Le rapport d'enseignants du collégial québécois à la lecture d'une œuvre complète : le plan didactique}

Julie Babin et Olivier Dezutter

\section{Les défis d'enseignement de la lecture d'une œuvre complète, d'un ordre d'enseignement à l'autre}

1 La lecture de l'œuvre littéraire complète (ci-après o.c.) s'inscrit dans une pratique sociale de référence que Baudelot et al. (1999) associent à la lecture «ordinaire», en dehors de contraintes scolaires. Cette lecture exige un temps certain (Daunais, 2000, Waszak et Dufays, 2015), notamment parce que, par définition, l'o.c. est un texte d'une certaine ampleur; il peut s'agir d'un texte suivi long (comme le roman) ou d'un assemblage de textes courts (comme le recueil de nouvelles). L'o.c. se caractérise par le fait d'être écrite avec une préoccupation esthétique, pensée par son auteur comme une unité (Aron et al., 2010) et publiée de concert avec l'éditeur'. Dans le cas d'œuvres romanesques ou dramatiques, accéder à cette unité exige chez le lecteur une construction de sens fondée sur des mises en relation complexes et des réévaluations constantes de l'apport de microéléments à la compréhension de l'ensemble du texte (Veck, 1998). Ces opérations cognitives se développent non seulement avec l'âge, mais aussi au fur et à mesure que se présentent de nouveaux défis de lecture (nouveaux genres, nouveaux contextes, nouvel univers narratif, etc.).

2 La lecture de l'o.c. fait de nos jours partie des exigences des programmes scolaires du primaire, du secondaire et du collégial. Dans ce contexte scolaire, Dezutter et al. (2005) décrivent l'o.c. comme celle dont l'enseignant « n'exploite pas que des extraits » (p. 85), et cette exploitation prévoit idéalement un accompagnement des élèves à trois moments clés : avant, pendant et après la lecture de l'ouvrage (Giasson, 2003). Le travail sur et 
autour de l'œuvre est souvent orienté autour d'un angle d'entrée dans le texte, que nous avons défini comme l'attention plus ou moins importante accordée à l'une ou l'autre des caractéristiques de l'œuvre (Babin, 2016). Bien que cet angle d'entrée soit déterminé (consciemment ou non) par le lecteur lors d'une lecture ordinaire, il n'en va pas de même lors des lectures scolaires. À l'école, l'angle d'entrée dans l'œuvre, que Renard (2007) nomme un « axe de lecture » est le plus souvent décidé par l'enseignant - ce - et ce, tant dans les classes du secondaire que dans celles du collégial (Babin, 2016, Dezutter et al., 2005, Renard, 2007, Waszak, 2017).

3 Pour une large proportion de jeunes québécois qui optent, après le secondaire, pour des études techniques ou préuniversitaires dans un établissement collégial (un cégep), le corpus d'œuvres imposé et le travail exigé sur celles-ci entrainera une rupture importante avec l'expérience scolaire vécue jusque-là${ }^{2}$. En effet, dans la large majorité des cégeps, le premier des trois cours de français obligatoires de 60 heures $^{3}$ (Gouv. du Québec, 2009) prescrit la lecture d'au moins deux œuvres de la littérature française du Moyen Âge au XVIIIe siècle. La lecture de ces œuvres privilégiée par le programme est axée surtout sur le repérage et l'interprétation des procédés stylistiques, et associée à la rédaction d'un texte de genre scolaire - l'analyse littéraire - s'apparentant au commentaire composé ( Ibid.). Plusieurs éditeurs proposent d'ailleurs des rééditions scolaires des œuvres, dont la version intégrale est complétée d'un appareillage didactique ciblant ces éléments formels. Les étudiants du collégial font donc face à des défis de lecture nouveaux. Ils sont aussi jugés faibles lecteurs par une majorité de leurs enseignants (Maisonneuve 2002). Ces derniers ont pour la plupart une formation universitaireen études littéraires, au sein de laquelle les enjeux didactiques de la lecture d'œuvres complètes par des lecteurs non experts sont peu ou pas abordés (Dezutter, 2009-2012) Dans ce contexte, nous avons souhaité mieux connaitre sous le plan didactique le rapport à la lecture d'une o.c. d'enseignants responsables du cours initial de français au collégial, intitulé Écriture et littérature. Parmi les deux plans du rapport à la lecture distingués par Émery Bruneau (2010) chez des futurs enseignants, à savoir le plan personnel et le plan didactique, nous nous intéressons donc ici au second plan.

\section{Le rapport à la lecture de l'œuvre littéraire complète}

\section{1 Les deux plans du rapport à la lecture}

4 Par rapport à d'autres propositions sur le rapport à l'écriture ou sur le rapport à l'écrit, la conceptualisation du rapport à la lecture littéraire d'Émery-Bruneau (2010) présente l'intérêt d'une prise en compte de la réalité des enseignants, réalité double en ce qu'il alimente un rapport tant personnel que didactique à la lecture littéraire. À cet égard, nous nous rangeons derrière cette définition, en ne la limitant toutefois pas à la forme spécifique d'appréhension du texte qu'est la lecture littéraire ; le rapport à la lecture de l'œuvre littéraire complète est défini comme un «ensemble diversifié de relations dynamiques d'un sujet-lecteur avec la lecture [...] constitué de deux plans» (Ibid., p. 57). Ces plans correspondent au rapport à la lecture de l'o.c. par la personne enseignante dans sa vie privée (plan personnel) d'une part et dans sa vie professionnelle (plan didactique) d'autre part. Le plan didactique comprend sa " prise en compte du [rapport à la lecture de cette o.c.] des élèves sujets-lecteurs et la façon dont [elle] entend les former " (plan didactique) (Émery-Bruneau, 2011, p. 38). Bien que lié au rapport personnel de 
l'enseignant, c'est à ce second plan que nous nous attardons plus particulièrement dans cette publication.

\section{2. Les quatre dimensions du rapport à la lecture}

5 Le rapport à la lecture d'un enseignant peut être examiné sous la loupe de quatre dimensions. L'analyse de la dimension épistémique permet d'accéder aux savoirs et aux savoir-faire liés à la lecture (nature et rôle) que l'enseignant conçoit nécessaires pour développer d'autres savoirs et savoir-faire. La dimension sociale correspond à la façon dont l'enseignant définit son rôle, celui des autres et celui de l'o.c. elle-même dans son « rapport au monde » comme lecteur ou dans celui des élèves. La dimension subjective, pour sa part, renvoie aux représentations et aux aspects " psychoaffectifs, axiologiques et réflexifs» (Ibid.) qui interviennent dans la lecture de l'o.c. Quant à la dimension praxéologique, elle permet de décrire les actions posées concrètement par l'enseignant autour de la lecture d'une o.c. dans son contexte d'enseignement: le choix de titres, la lecture qu'il fait des o.c. et qu'il impose aux étudiants, les tâches qui l'entourent, etc.

\section{3. Les cinq angles d'entrée dans la lecture d'une œuvre complète}

6 Selon Dufays et al. (2009) «[1]e rapport à la littérature mobilise par sa nature même la lucidité et l'illusion, la réflexion et la détente, la distanciation et la participation" (Dufays et al., 2009, p. 6), le choix d'angle d'entrée dans l'œuvre fait par l'enseignant orientera inévitablement de préférence les élèves vers une lecture distanciée ou participative, ou vers une démarche qui amène à combiner les deux et qui est considérée comme révélatrice de la lecture littéraire d'une œuvre (Ibid.).

7 Selon le cadre que nous avons construit, trois angles convoquent plus particulièrement une lecture de distanciation, à savoir les angles esthétique, institutionnel et historique. Le premier s'avère d'ailleurs l'un des plus spontanément associés aux études littéraires. Plusieurs auteurs s'entendent en ce sens sur le fait que l'œuvre littéraire se caractérise par un traitement particulier du langage (Aron et Viala, 2005, Falardeau, 2003, Rouxel, 2004) qui procure au lecteur une expérience en relation avec ce dernier (Larue, 2007). Le deuxième angle, institutionnel, renvoie au jugement porté sur l'œuvre par différentes instances pour la qualifier de littéraire, en fonction de son "appartenance à des structures institutionnelles et de la valeur symbolique qui [lui] est attribuée » (Falardeau, 2003, p. 674). Cette inscription de l'œuvre dans le champ littéraire va souvent de pair avec une lecture sous un troisième angle, historique, au cours duquel l'attention du lecteur est dirigée vers le lien à établir entre le passé et le présent (Ibid.). L'œuvre assurant une communication différée dans le temps (Aron et Viala, 2005) entre le moment d'écriture et le moment de lecture, le contexte sociohistorique dans lequel elle a été produite (Legros, 1998, 2008) offre au lecteur un regard complémentaire sur le sens du texte.

8 Les angles subjectif et formateur motivent davantage une lecture de participation. L'œuvre littéraire, abordée sous l'angle subjectif, est vue comme un reflet unique de l'expérience humaine (Falardeau, 2003) qui permet au lecteur de s'ouvrir à la sensibilité de l'auteur et à son interprétation du monde (Todorov 2007). Pour Langlade et Fourtanier (2007), d'ailleurs, cette "implication du lecteur dans l'œuvre apparait comme une nécessité fonctionnelle de la lecture littéraire» (p. 103), au cours de laquelle le lecteur accède à l'universalité contenue dans l'œuvre sans le risque de se compromettre lui- 
même sur le plan individuel (Adam et Cordonier, 1998). Partant, l'œuvre littéraire peut être abordée sous l'angle formateur, qui consiste à donner l'occasion au lecteur de produire une pensée complexe lui permettant d'apprendre et de se former comme individu (Legros, 1998), qui participe à sa quête personnelle de sens (LARUE, 2007) et qui alimente son imaginaire (Adam et Cordonier, 1998).

Le choix didactique d'un angle d'entrée dans l'œuvre plutôt qu'un autre nous parait non seulement relever de la dimension praxéologique du rapport à la lecture de l'o.c., mais être aussi intrinsèquement lié aux autres dimensions du rapport qu'entretient l'enseignant à cette lecture. En effet, il nous semble que l'enseignant privilégiera tel ou tel angle selon la façon dont il conçoit ces angles et les savoirs nécessaires au développement de la compétence à lire (dimension épistémique), selon la valeur qu'il accorde à chacun des angles dans son enseignement et au rôle qu'il se donne dans le développement du rapport au monde des étudiants (dimension sociale), selon l'écho qu'à l'œuvre sur lui et ce qu'il veut faire ressentir aux étudiants lorsqu'il en est question (dimension subjective), et selon sa façon d'enseigner ou les activités qu'il met en œuvre (dimension praxéologique).

\section{4. Les orientations du programme ministériel}

L'angle de lecture pourrait être déterminé par les étudiants du collégial eux-mêmes, mais le programme d'études balisant le cours d'Écriture et littérature engage plus ou moins explicitement les enseignants à leur proposer une lecture des œuvres sous des angles soutenant surtout une lecture de distanciation.

11 Le choix des œuvres doit d'abord être guidé en partie par leur potentiel de lecture d'un point de vue historique ou institutionnel ; les œuvres à faire lire aux cégépiens devraient être celles qui "ont marqué l'histoire de la littérature " (Gouv. du Québec, 2009). De surcroit, une lecture sous l'angle esthétique est explicitement encouragée, non seulement par l'attention à porter aux " procédés d'écriture » mais aussi par la « pratique régulière de la lecture et de l'écriture ", qui conduirait les élèves à "développer une sensibilité esthétique " (Ibid.). Selon l'interprétation donnée à cette dernière prescription, certains enseignants pourraient également exploiter un angle d'entrée plus subjectif, mais les références ministérielles à une telle lecture de participation sont limitées. Néanmoins, un angle formateur pourrait être adopté par certains enseignants qui souhaitent développer une attitude d'« ouverture à d'autres cultures et au monde par la lecture d'œuvres littéraires [et permettre aux étudiants de] saisir les enjeux sociaux, par l'analyse de diverses représentations du monde » mises de l'avant dans ces œuvres (Ibid.).

En tenant compte du contexte institutionnel présenté et selon les cadres que nous avons retenus, nous avons donc cherché à mieux comprendre comment des enseignants du collégial qui ont suivi une formation initiale - dans laquelle la formation didactique est peu ou pas du tout présente - organisent la lecture des œuvres littéraires complètes, quels sont les angles d'entrée dans les œuvres qu'ils privilégient et ce que ces choix révèlent de leur rapport à la lecture des œuvres sur le plan didactique. 


\section{Méthodologie} la lecture d'œuvres littéraires complètes : analyse de plans de cours, questionnaire en ligne, entretiens semi-dirigés et analyse de leçons filmées (Dezutter et al., 2009-2012). Nous proposons ici une analyse secondaire de données qualitatives collectées dans les deux dernières phases, auprès de trois enseignants volontaires du collégial: Esther, Martin et François ${ }^{4}$. Les résultats présentés ici émanent en partie d'une recherche doctorale (Babin, 2016), au cours de laquelle a été réalisée une analyse thématique (Paille et Mucchielli 2010) du propos des enseignants lors de leçons filmées à l'automne 2012 afin de déterminer les angles d'entrée privilégiés par chacun. Ces leçons, organisées en une séquence d'enseignement, portent, dans le cas d'Esther, sur Micromégas ( $\pm 11 \mathrm{~h}$ de travail de classe), dans le cas de Martin, sur Le Mariage de Figaro $( \pm 8,5 \mathrm{~h})$ et dans celui de François, sur Dom Juan $( \pm 14,5 \mathrm{~h})$. Afin d'apporter un éclairage nouveau à ces résultats, nous avons reconduit l'analyse thématique réalisée par Babin (2016) sur des données qui n'avaient pas été traitées dans le cadre de la recherche de Dezutter et al. (2009-2012), à savoir les propos tenus par chacun de ces enseignants lors de l'entretien semi-dirigé. Les extraits colligés au terme de l'analyse ont fait émerger une description - sur le plan didactique du rapport à la lecture de l'o.c. de chacun au regard des quatre dimensions qui le composent.

\section{Des rapports uniques à la lecture de l'œuvre complète}

Comme le rapport à l'écrit, le rapport à la lecture d'œuvres complètes est propre à chaque individu ; trois portraits distincts seront donc dressés afin de montrer les spécificités, sur le plan didactique, de ce rapport pour chacun des enseignants. Il convient néanmoins de souligner que les trois entretiens révèlent la nette distinction faite par les enseignants entre le plan didactique et le plan personnel, où la lecture est implicitement moins en profondeur. Cette différence est par exemple observable dans la lecture attendue des étudiants : ces derniers doivent « creuser le texte » (François), « aller plus loin » (Martin) et, selon Esther, "une bonne partie des étudiants faibles" ne peuvent pas y arriver, même au prix de grands efforts. Les trois enseignants partagent aussi un même point de vue sur le choix des œuvres : ils qualifient tous certaines œuvres d' " extraordinairement bien écrites » (François), de "géniales » (Esther), mais avouent du même souffle que c'est «beaucoup trop dur à aborder [...] beaucoup trop long et difficile » pour des étudiants d'Écriture et littérature (Martin). L'appréciation personnelle ne suffit donc pas à retenir un titre plutôt qu'un autre ni à imposer tel ou tel travail de classe ; des enjeux didactiques émanant des dimensions sociale, épistémique et subjective s'ajoutent à la réflexion.

\section{1 Martin : lire pour analyser}

Martin n'a qu'une année d'expérience au moment de la collecte de données, et ce statut professionnel précaire semble teinter plusieurs décisions entourant la séquence sur Le Mariage de Figaro, décisions qui nous incitent à décrire son rapport à la lecture de l'o.c. comme une "valse" entre le respect strict de contraintes ministérielles et une 
conception plus humaniste de la littérature. Concernant la dimension praxéologique par exemple, bien qu'il dise organiser sa séquence autour du comique pour tenter d'entrée dans l'œuvre sous l'angle subjectif, il est dans les faits porté à aborder davantage en classe les procédés d'écriture - notamment l'ironie - comme le prescrit le programme d'études. Il adopte ainsi un angle esthétique en vue de faire rédiger aux étudiants une analyse littéraire, dans laquelle ces derniers devront « montre[r], à l'aide des extraits choisis, que [l'œuvre] est une critique sociale» (cours 5; 00:05:45). Pour ce faire, Martin se trouve contraint d'adopter aussi un angle historique qui permettra aux étudiants de comprendre le contexte de Beaumarchais et de se positionner, lors de la rédaction évaluée, sur la dimension critique de l'o.c. Or, pour y arriver, les étudiants doivent avoir compris le propos, et comme les activités ne commencent qu'après la lecture autonome de l'œuvre en dehors des cours, Martin s'assure de la compréhension du texte par la paraphrase systématique de tous les extraits traités en classe en s'appuyant régulièrement sur l'objetlivre. Il organise aussi une lecture à l'italienne et le repérage en équipe de passages ironiques (cours 2). Plusieurs exercices sont inspirés des annexes à l'édition scolaire de l'œuvre achetée (Bourdonnais, 2001).

Le portrait qui précède s'éclaire sous l'analyse de la dimension épistémique. Pour Martin, un certain nombre de savoirs liés à la langue semblent nécessaires à la compréhension des o.c. imposées. Il révèle par exemple lors de l'entretien que le «vocabulaire plus ancien » peut poser problème à certains étudiants, mais aussi « des mots vraiment de base ", d'où peut-être son recours régulier à la paraphrase. Dans le même sens, un minimum de savoirs relatifs à l'histoire lui parait incontournable; il s'étonne du peu de " connaissances historiques» des étudiants. Il pallie donc ce manque par des exposés pour s'assurer que ceux-ci disposent de l'essentiel lors de l'évaluation: «Une fois dans l'analyse, ils peuvent [...] réutiliser [les informations sur le contexte] et ça peut les orienter ». De l'avis de Martin, l'écriture de l'analyse, parce qu'elle exige une certaine structure, peut soutenir la compréhension de l'organisation globale d'autres textes, notamment celle des o.c. lues.

La dimension sociale explique de manière encore plus évidente le va-et-vient entre les différents angles d'entrée. D'une part, Martin confie la place importante que prend le programme d'études dans le traitement des o.c. en classe - en particulier au regard des figures de style et de l'écriture de textes scolaires comme la dissertation ou l'analyse: " s'il fallait respecter juste [le programme], on ne s'y intéresserait pas du tout [au propos et au contexte sociohistorique de l'œuvre], mais on s'attarderait vraiment à une vision formaliste de la chose ». D'autre part, il souhaite avoir le rôle, au collégial, d'« ouvrir les horizons de ces élèves-là, [de leur donner] une ouverture sur le monde, une compréhension du monde par les œuvres littéraires [...] une compréhension de l'être humain aussi » et de les outiller pour lire n'importe quel texte dans le futur. Pour l'enseignant, l'o.c. offre plusieurs voies interprétatives et il lui parait «très important qu'il y ait de l'espace » en classe pour d'autres interprétations que la sienne, « même dans le cadre très serré » du cours. Il prévoit pour cela des moments de discussion entre étudiants et valorise le travail d'équipe.

Enfin, le discours de Martin nous renseigne sur la dimension subjective de son rapport à la lecture de l'o.c., laquelle s'articule selon la même logique que les autres dimensions : si l'enseignant trouve essentiel de choisir une œuvre qu'il aime lui-même et d'être proche de l'intérêt des jeunes de sa classe, s'il considère qu'une pièce de théâtre pourrait réconcilier certains d'entre eux avec la lecture, il avoue par ailleurs que «les figures de 
style, c'est le lot » de l'enseignant au collégial et que le programme d'études « en arrive malheureusement à une instrumentalisation » de la littérature qui « ne permet pas d'être dans une interprétation de l'œuvre ».

\section{2 François : lire pour comprendre le comportement humain}

19 François enseigne le français au collégial depuis une vingtaine d'années. Sur le plan didactique, son rapport à la lecture d'une o.c. s'explique avant tout par le choix d"impliquer concrètement les étudiants dans la réflexion sur Dom Juan. En effet, au regard de la dimension praxéologique, François remet d'abord un questionnaire d'accompagnement à la lecture, où la moitié des items ( 9 sur 18) vont en ce sens ${ }^{5}$. L'enseignant encourage donc un angle d'entrée subjectif dans l'œuvre, par lequel les étudiants sont amenés, au terme de la lecture de la tragicomédie, à juger de la moralité du personnage principal au cours d'un procès simulé. Ils doivent y exercer leur jugement en confrontant leur point de vue d'aujourd'hui à celui des contemporains du personnage principal, notamment à l'égard du mensonge ou des rapports entre hommes et femmes. L'objet-livre constamment en main, François dirige ses étudiants vers une lecture parallèle de la pièce sous l'angle historique, solidement arrimé à l'angle subjectif dans la mesure où il y intègre surtout des thématiques sociales qui suscitent la prise de position, comme le libertinage, la religion et l'honneur. Ces entrées dans Dom Juan s'expliquent vraisemblablement par des éléments relevant de la dimension subjective du rapport à la lecture de l'o.c. de l'enseignant. Il est en effet raisonnable de penser que son choix d'œuvres pour la classe s'appuie sur ses propres critères de sélection: le professeur dit retenir des titres qui mettent en évidence l'universalité des phénomènes humains malgré l'époque à laquelle elles ont été publiées et qui permettent de se retrouver dans l'œuvre.

Outre l'importance que François dit accorder à la culture générale pour comprendre une o.c., sa préoccupation pour les savoirs historiques n'apparait pas clairement dans l'analyse de la dimension épistémique. Dans l'entretien, il parle surtout des savoirs liés au langage et à la forme des œuvres pour comprendre ces dernières. Écriture et littérature lui parait "un cours assez ardu», où les o.c. prescrites engagent des défis plus importants que les extraits en raison de leur ampleur. Il se rend « compte qu'il vaut mieux aller dans [...] des petits passages plutôt que parler, dans l'ensemble, de l'œuvre parce qu'ils [les étudiants] se perdent ». La lecture de l'o.c. nécessite donc des savoir-faire qui lui sont propres, comme la détermination d'un lieu et d'un moment appropriés de lecture et une certaine concentration de la part des étudiants, qui pourtant : "lisent ça en autobus ou entre deux activités, un paragraphe ici et là. [...] Leur attention est extrêmement dispersée ». La maitrise de la citation parait aussi essentielle pour soutenir une argumentation sur le texte lu : pour l'enseignant, le but de la lecture est non seulement de le comprendre, mais aussi de le critiquer. En ce sens, le procès s'avère un choix d'activité cohérent.

21 Au regard de la dimension sociale, François affirme à l'égard du programme : «j'essaie de pas trop me fier là-dessus ». Il considère surtout que les enseignants du collégial doivent amener les étudiants à structurer et à " articuler une pensée ", à faire des liens entre l'o.c. et «leur monde à eux », ce qui soutient sans doute des apprentissages sur le monde qui les entoure. Pour ce faire, il reconnait la valeur de la discussion entre pairs, où les étudiants «entendent les autres réponses» et doivent s'ajuster, ce qui motive 
probablement son choix d'organiser des exercices oraux comme des ateliers, des tables rondes ou un procès comme celui de Dom Juan.

\section{3 Esther : lire pour écrire}

Le rapport d'Esther à la lecture de l'o.c. en contexte scolaire prend vraiment sens si l'on s'appuie sur la prémisse qu'elle aborde l'activité lectorale de son point de vue d'écrivaine : « je suis moins lectrice que quelqu'un qui écrit », dit-elle. Un certain nombre de décisions relevant de la dimension praxéologique s'inscrivent d'ailleurs en ce sens. Sa séquence d'enseignement autour de Micromégas, par exemple, mène les étudiants à la rédaction d'un chapitre supplémentaire à l'œuvre, sous la forme du pastiche. Pour les y préparer, après que les étudiants ont lu la lecture de manière autonome, l'enseignante fait le choix d'entrer dans l'œuvre principalement sous l'angle esthétique, en mettant à profit des moments d'observation de la forme, que ce soit lors d'un exposé sur les caractéristiques génériques du conte au cours 1 ou d'une activité d'imitation de la forme syntaxique des titres de chapitres au cours 3. Néanmoins, du point de vue strict de la lecture, cet angle d'entrée dans l'œuvre n'est explicitement identifiable au total que durant une vingtaine de minutes (sur $11 \mathrm{~h}$ filmées) : le plus souvent, ces aspects formels sont convoqués en vue de la tâche d'écriture. L'objet livre lui-même est remplacé en tout temps par des extraits recopiés dans un diaporama et les questions que pose Esther sont parfois tirées de l'appareillage didactique en annexe à l'o.c. La priorité accordée à l'activité d'écriture s'observe aussi par l'angle subjectif adopté pour entrer dans le conte de Voltaire. Le plus souvent, l'impact affectif de l'œuvre sur les lecteurs que sont les étudiants est occulté par les émotions à susciter comme scripteurs du pastiche, en particulier la stupéfaction et l'amusement. L'enseignante met par exemple en garde une étudiante: «Tu vas y penser, à m'amuser? Pour que ce soit amusant?» (cours 5; 00:13:13).

Les choix qui précèdent semblent s'expliquer en partie par les autres dimensions du rapport d'Esther à la lecture de l'œuvre sur le plan didactique. En ce qui a trait à la dimension épistémique, par exemple, lecture et écriture sont intimement liées: l'apprentissage de la dissertation, pour l'enseignante, «c'est de la littérature parce que c'est de l'analyse ». Son discours lors de l'entretien amalgame également lecture et littérature; si lire lui parait une épreuve pour ses étudiants, il faut ainsi entendre que c'est la lecture - analytique - de la littérature qui leur pose problème. En réponse à cette difficulté, lorsqu'elle est interrogée sur les stratégies de lecture, elle parle plutôt de "stratégie de travail ", qu'elle ne précise pas. En cohérence avec cette posture ancrée dans les études littéraires, elle affirme que la lecture de textes littéraires variés, qu'il s'agisse ou non d'œuvres complètes, permet d'acquérir des savoirs sur la langue et la littérature.

$\mathrm{Au}$ regard de la dimension sociale, Esther considère que les enseignants du collégial ont pour « mission » d'initier les jeunes adultes à la littérature, de leur donner le gout de lire au-delà du milieu scolaire, de développer leur ouverture sur le monde et de créer des citoyens de demain. Or, elle juge que le programme d'études va à l'encontre de ce mandat en contraignant les enseignants à aborder les œuvres d'abord et avant tout du point de vue formel, ce qui explique peut-être en partie son choix d'angle esthétique. Cette perspective va de pair avec plusieurs éléments relevant de la dimension subjective du rapport à la lecture des œuvres : celle-ci est une rencontre, une occasion d'introspection 
pour le lecteur dans la mesure où elle fait écho à ses préoccupations. Convaincue que les titres imposés, parce qu'ils sont des chefs d'œuvre, sont faciles à aimer, Esther affirme que leur lecture n'est néanmoins possible que si les étudiants ont les capacités préalables, s'ils aiment déjà lire, bref s'ils « viennent de bonnes familles ». Tout en exprimant son besoin d'une formation en didactique de la lecture, l'enseignante souhaite avec cynisme que les œuvres proposées rejoignent ses étudiants un tant soit peu et que ces derniers se donnent au moins la peine de « lire le résumé sur internet ».

\section{Discussion}

L'analyse réalisée à partir des cas de Martin, de François et d'Esther montre l'unicité de leur rapport à la lecture d'une o.c. sur le plan didactique. Pourtant, une lecture transversale des quatre dimensions dudit rapport met en évidence des constantes. Les résultats relatifs à la dimension praxéologique, par exemple, permettent de montrer l'écart entre ce qui caractérise la lecture d'une o.c. comme pratique sociale de référence. Si les étudiants font, dans les faits, une lecture intégrale, cela ne signifie pas qu'est réalisé en classe un travail sur l'entièreté de l'œuvre. À contrario de la définition de l'œuvre complète proposée par Dezutter et al. (2005), il semble bien que les enseignants n'exploitent que des extraits, et que les particularités des liens entre extraits et structure globale propres à une lecture intégrale (Veck, 1998) relèvent de la responsabilité des lecteurs, apprentis, rappelons-le. De surcroit, malgré les difficultés que les étudiants du cégep rencontrent, deux enseignants sur trois n'offrent aucune préparation ni aucun accompagnement à la lecture, deux temps pourtant déterminants dans la lecture d'une o.c. (Giasson, 2003). Enfin, comme nous l'avons démontré précédemment à l'instar d'autres auteurs (Babin, 2016, Renard, 2007, Waszak, 2017), l'angle d'entrée retenu par Martin, par François ou par Esther n'est pas celui des étudiants, ce qui serait le cas si ces derniers lisaient les mêmes classiques par eux-mêmes en contexte extrascolaire.

En ce qui a trait à la dimension épistémique, Martin et François partagent l'avis que des savoirs sur la langue et des savoirs sur la macrostructure de l'œuvre sont nécessaires pour comprendre cette dernière, ce qui est déjà reconnu (Falardeau, 2003, Giasson, 2003). Pour Esther, le processus est perçu inversement : c'est la lecture de l'o.c. qui permettrait d'acquérir de tels savoirs. Elle évoque en cela l'intérêt formateur de toute œuvre littéraire (Babin, 2016). Dans le même sens, le fait que les enseignants valorisent tous trois le choix d'une o.c. où les jeunes se retrouvent et qui fait écho à leur vie personnelle constitue le principal point commun relatif à la dimension subjective.

Au regard de la dimension sociale, le rapport des enseignants ciblés par notre analyse à la lecture de l'o.c. est inévitablement influencé par la place du programme d'études, sur le plan didactique. À cet égard, les exigences du Programme de formation générale commune (Gouv. de Québec, 2009) paraissent dicter l'adoption, dans une plus ou moins grande mesure selon l'enseignant, d'un angle d'entrée esthétique dans l'œuvre. Si Martin et Esther considèrent cette prescription comme une contrainte qui balise leurs décisions, François en parle avec philosophie et déclare en tenir compte avec un certain détachement. Nonobstant, les trois enseignants mentionnent le rôle important de la lecture de l'œuvre littéraire complète dans l'ouverture sur le monde, un rôle que plusieurs écrits confirment (Adam et Cordonier, 1998, Falardeau, 2003, Todorov 2007), mais dont les Martin et Esther n'arrivent que peu, à terme, à convaincre les étudiants de leur classe. 
Au final, l'analyse du rapport à la lecture d'une o.c de trois en études collégiales permet d'une part de poser un regard sur les limites des prescriptions officielles et de relever le poids des épreuves d'évaluation centrées sur le commentaire de texte. D'autre part, les analyses effectuées nous amènent à penser que le cadre relatif aux différentes dimensions du rapport à la lecture d'une œuvre complète pourrait constituer également un outil à exploiter dans un contexte de formation initiale ou continue des enseignants du collégial à la didactique de la lecture littéraire.

\section{BIBLIOGRAPHIE}

Adam (J.-M.) et Cordonier (N.), « Enseigner la littérature aujourd'hui », Le Français aujourd'hui, vol. 121, pp. 6-11, 1998.

Aron (P.), Saint-Jacques (D.) et Viala (A.), Le dictionnaire du littéraire. $3^{\mathrm{e}}$ éd., Paris : Presses universitaires de France, 2010, 814 p.

Aron (P.) et Viala (A.), L'enseignement littéraire. Paris : Presses universitaires de France, 2005, 128 p.

Babin (J.), La lecture d'œuvres littéraires complètes au collégial : des cas de pratiques d'enseignement dans le cours d'Écriture et Littérature (601-101). éducation. Sherbrooke (QC): Université de Sherbrooke, 2016, 475 p.

Baudelot (C.), Cartier (M.) et Detrez (C.), Et pourtant, ils lisent... Paris, Seuil, 1999, 245 p.

Bourdonnais (G.) (dir.), Le Mariage de Figaro de Beaumarchais - Étude de l'œuvre. Laval (QC), Groupe Beauchemin éditeur (coll. « Parcours d'une œuvre »), 2001.

Daunais (I.), « Une vitesse littéraire : la lenteur », L’inconvénient : revue littéraire d'essai et de création vol. 1, pp. 7-19, 2000.

Dezutter (O.), La place des œuvres complètes dans l'enseignement du français au collégial, CRSH, no 410-2009-1644, 2009-2012.

Dezutter (O.), Morissette (C.), Bergeron (M.-D.) et Larivière (I.), « Quel programme de lecture pour les élèves québécois de 10 à 17 ans ? ». Québec français, vol. 139, pp. 83-85, 2005.

Dufays (J.-L.), Lisse (M.) et Meurée (C.), Théorie de la littérature : Une introduction. Louvain-la-Neuve, Belgique : Academia-Bruylant. 2009, 208 p.

Émery-Bruneau (J.), Le rapport à la lecture littéraire : des pratiques et des conceptions de sujetslecteurs en formation à l'enseignement du français à des intentions didactiques. Québec :

Université Laval, 2010, 432 p.

Émery-Bruneau (J.), « La dimension sociale du rapport à la lecture littéraire d'étudiants en enseignement : un indice du développement de l'identité professionnelle », Revue canadienne de l'é ducation, vol. 34, no 2, pp. 34-52, 2011.

Émery-Bruneau (J.), «Compréhension et interprétation : deux composantes complémentaires de la lecture littéraire », Revue des sciences de l'éducation, vol. 29, no 3, pp. 673-694, 2003. 
Giasson (J.), La lecture : de la théorie à la pratique. $2^{\mathrm{e}}$ éd., Boucherville, QC : Gaëtan Morin éditeur, 2003, $398 \mathrm{p}$.

Gouv. du Québec, Formation générale commune, propre et complémentaire aux programmes d'études conduisant au diplôme d'études collégiales. Québec, QC : Ministère de l'Éducation, du Loisir et du Sport, 2009.

Langlade (G.) et Fourtanier (M.-J.), « La question du sujet lecteur en didactique de la lecture littéraire», in Falardeau, E., Fisher, C., Simard, C. et Sorin, N. (Ed.), La didactique du français - Les voies actuelles de la recherche. Québec : Les Presses de l'Université Laval, 2007.

Larue (M.), De fil en aiguille : essais, Montréal : Boréal, 2007.

Legros (G.), « Enseigner aujourd'hui la littérature », Lecteurs de littérature - Le français aujourd'hui, vol. 121, pp. 12-17, 1998.

Legros (G.), « Quelle place pour la didactique de la littérature », in Chiss, J.-L., David et Reuter, Y. (Ed.), Didactique du français. Fondements d'une discipline. Bruxelles : De Boeck, 2008.

Maisonneuve (L.), Le cégépien lecteur : étude des perceptions et des représentations de la lecture ainsi que des attitudes envers les pratiques scolaire et personnelle de la lecture et analyse des lectures effectives sciences de l'éducation. Montréal : Université du Québec à Montréal, 2002.

Paillé (P.) et Mucchielle (A.), L'analyse qualitative en sciences humaines et sociales, $2^{\mathrm{e}}$ éd. Paris : Armand Colin, 2010, 315 p.

Renard (F.), Les Lectures scolaires et extra-scolaires de lycéens : entre habitudes constituées et sollicitations contextuelles. Lyon, France : Université Lumière Lyon 2, 2007, 1074 p.

Rouxel (A.), «Qu'entend-on par lecture littéraire ? », in Tauveron, C., La lecture et la culture litté raires au cycle des approfondissements. Paris : SCERÉN, 2004.

Schneuwly (B.) et Dolz, (J.). Les genres scolaires, des pratiques langagières aux objets d'enseignement. Repères, vol. 15, pp. 27-40, 1997.

Todorov (T.), La littérature en péril, Paris : Flammarion, 2007, 94 p.

Veck (B.). CEuvres intégrales et projet de lecture, Paris : INRP - Bertrand-Lacoste, 1998, 223 p.

Waszak (C.), Entre contextualisation et actualisation, quelle lecture des romans du XIX ${ }^{\mathrm{e}}$ siècle à la fin du secondaire ? État des lieux et analyse des dispositifs didactiques. Louvain-la-Neuve : Université catholique de Louvain, 2017.

Waszak (C.), et Dufays (J.-L.), « La lecture de romans du XIX ${ }^{\mathrm{e}}$ siècle au secondaire - analyse de pratiques enseignantes ", in Dezutter, O. et Falardeau, E. (Ed.), Les temps et les lieux de la lecture. Namur: Presses universitaires de Namur - CEDOCEF, 2015.

\section{NOTES}

1. Notons néanmoins que les technologies numériques permettent maintenant aux auteurs de publier eux-mêmes leurs textes, ce qui tend à faire évoluer la définition de l'o.c., en particulier au regard des recueils.

2. Dezutter et al. (2005) ont établi que les élèves du secondaire lisent en moyenne cinq o.c. par année, le plus souvent des romans issus de la littérature québécoise contemporaine ou de la littérature traduite. Une majorité d'enseignants vérifient cette lecture en exigeant un résumé ou en imposant un questionnaire sur le contenu de l'œuvre.

3. Chaque cours est d'une durée de 15 semaines. 
4. Des prénoms fictifs ont été attribués pour protéger l'anonymat.

5. Les autres items portent sur des éléments de structure de la pièce (exposition, nœud, dénouement, règle des trois unités, etc.).

\section{RÉSUMÉS}

Les premiers cours de français obligatoires au collégial exigent pour une majorité d'étudiants la lecture intégrale d'œuvres littéraires qu'ils ont peu fréquentées jusque-là. Il s'agit d'un défi pour les enseignants, pour la plupart peu formés en didactique. Pour mieux comprendre leur rapport à la lecture d'une œuvre sur le plan didactique, une étude multicas $(n=3)$ a été réalisée. L'analyse de pratiques de classe et d'un entretien montre une tension entre prescriptions du programme et vision du rôle de la lecture scolaire d'une œuvre littéraire, qui engage des pratiques variées.

The first collegial course requires most of the students to read whole literary works they have never been in contact with. Because of the limited background teachers have in didactics, they often meet challenges supporting them. To better understand the relationship these teachers have to such literary works full reading, we have carried out a multicase study $(n=3)$. Class practices and teachers interviews analyzed essentially show a tension between core prescriptions and teachers' vision of the role of reading a whole literary work in class, leading to a range of practices.

\section{INDEX}

Keywords : literary masterpiece, relationship to reading, teaching, cegep

Mots-clés : œuvre complète, rapport à la lecture, enseignement, cégep

\section{AUTEURS}

\section{JULIE BABIN}

Ph. D., chargée de cours

\section{OLIVIER DEZUTTER}

Professeur titulaire et codirecteur du Collectif de recherche CLÉ, Université de Sherbrooke 